8 Research Square

\title{
Assessing COVID-19 related anxiety among nurses in Malawi
}

Genesis Chorwe-Sungani ( $\nabla$ genesischorwe@kcn.unima.mw )

Kamuzu College of Nursing, University of Malawi https://orcid.org/0000-0002-4296-6394

\section{Research Article}

Keywords: Anxiety, COVID-19, Nurse, Prevalence, functional impairment

Posted Date: September 18th, 2020

DOI: https://doi.org/10.21203/rs.3.rs-79619/v1

License: (a) (i) This work is licensed under a Creative Commons Attribution 4.0 International License. Read Full License 


\section{Abstract}

Introduction: Psychological wellbeing of nurses is crucial for them to effectively discharge their duties. However, COVID-19 related anxiety can interfere with nurses' performance in their duties and reduce their self-efficacy levels.

Aim: This study aimed at assessing COVID-19 related anxiety among nurses in Malawi.

Method: This was a cross-sectional study that collected data from 102 nurses online. Data were analysed using descriptive statistics and receiver operating curve analysis.

Results: This study found that $25.5 \%(n=26)$ of respondents had COVID-19 related anxiety and $48 \%$ $(n=49)$ functional impairment. There were significant differences in the numbers of respondents who had functional impairment in relation to workplace $\left(x^{2}=8.7, p=0.03\right)$ with many of those working in hospitals $(58.6 \%, n=34)$ having highest levels (Mean $=20.6 \pm 10.4)$. The Coronavirus Anxiety Scale proved to be an effective instrument (Sensitivity $=73.1 \%$; Specificity $=60.5 \%$; AUC $=0.73$ ) for assessing COVID-19 related anxiety among nurses.

Discussion: Prevalence of COVID-19 related anxiety is high among nurses and many of them experience functional impairment. The CAS should be used in clinical practice for detecting COVID-19 related anxiety among nurses.

\section{Implications for practice}

Nurses should be screened of COVID-19 related anxiety using valid instrument. Psychosocial interventions should be part of preparedness to reduce COVID-19 impact on nurses.

\section{Accessible Summary}

\section{What is known on the subject?}

- Nurses as front-line health care workers are vulnerable to negative mental health effects from COVID19.

- Nurses are more likely to experience anxiety symptoms related to COVID-19 than other health workers.

\section{What the paper adds to existing knowledge?}

- One in every four nurses suffer from COVID-19 related anxiety.

- The nurses who work in hospitals are at high risk of COVID-19 related anxiety compared to those who work in Non-Governmental Organisation

- One out of two nurses suffer from functional impairment related to COVID-19. 
- The Coronavirus Anxiety Scale is a reliable instrument that can effectively detect COVID-19 related anxiety among nurses.

\section{What are the implications for practice?}

- Nurses should be screened of COVID-19 related anxiety using a valid instrument.

- Psychosocial interventions targeting nurses should be a part of preparedness to reduce COVID-19 impact on their well-being and the health system at large.

\section{THE RELEVANCE OF THEIR PAPER TO MENTAL HEALTH NURSING}

This paper is relevant to mental health nursing because it is the first one to document prevalence of COVID-19 related anxiety among nurses in Malawi. It has also validated the Coronavirus Anxiety Scale in the local setting. As such mental health nurses will have an effective instrument that they can use to screen for COVID-19 related anxiety among fellow nurses working in various settings. The paper also provides policy makers with information they can utilise when formulating or implementing nursing interventions during this COVID-19 pandemic.

\section{Introduction}

COVID-19 is fast spreading across the globe causing an outbreak. Health care professionals from various disciplines and cadres are involved in the care of patients with COVID-19 (Shanafelt, Ripp, \& Trockel, 2020). The high prevalence of COVID-19, its novelty and rapid spread, and the associated morbidity and mortality rates have acutely overstretched the limits of health care systems worldwide (Maben \& Bridges, 2020). This is a situation in Malawi where health care services have been overwhelmed from the first time a case of COVID-19 was detected on $2^{\text {nd }}$ April 2020. Data from Public Health Institute of Malawi reported by media indicate that as of September 2020, more than 5500 people have tested positive of COVID-19 and the numbers keep on rising. Additionally, more than 150 people including one health worker have died of the disease in Malawi. Anecdotal reports from Ministry of Health indicate that there are 67 nurses who have tested positive for COVID-19 in Malawi.

The upsurge of COVID-19 patients has caused an increase in care demands on nurses both in hospitals and community (Maben \& Bridges, 2020). Consequently, it has negatively affected some nurses psychologically. It is documented that nurses are now afraid of going to work because they fear for their lives and family (Nelson \& Lee-Winn, 2020). They suffer from great amount of anxiety every time they go into work because they fear that they will get sick of COVID-19 and end up dying like their patients (Nelson \& Lee-Winn, 2020). The high infection and mortality rates related to COVID-19 cause extensive fear and anxiety (Ahorsu et al., 2020). As such, identifying high-risk groups for psychological symptoms is as important as recognizing the presence of these symptoms, as they will be the target populations for evaluation and perhaps treatment (Evren, Evren, Dalbudak, Topcu, \& Kutlu, 2020). Literature indicate that 
front line health care workers are particularly vulnerable to negative mental health effects from COVID-19 (Wong, Pacella-LaBarbara, Ray, Ranney, \& Chang, 2020).

In Malawi, nurses constitute the bulk of frontline health care workers who are fighting the COVID-19 outbreak. However, these nurses among other challenges are faced with increased workload and threat of COVID 19 which may aggravate psychological pressure they experience. Some nurses have lamented that one of the hardest things to cope with during the COVID-19 pandemic has been the inconsistencies and constant changes (Nelson \& Lee-Winn, 2020) they experience in their work places. For instance, conflicting and rapidly changing information about personal protective equipment exacerbates health care workers' ongoing fears of exposure and uncertainty about their own safety in the workplace (Wong et al., 2020). Furthermore, protocols for procedures keep on changing rapidly, causing anxiety about whether what they used to do prior to such changes was adequate for themselves and patients (Nelson \& Lee-Winn, 2020). This shows that nurses and other people who spend a lot of time thinking about pandemics are at the highest risk for mental illness (Evren et al., 2020) including anxiety.

A systematic review reported a pooled prevalence of COVID-19 related anxiety among health workers as 23.2\% (Pappa et al., 2020). In Nepal, nurses were found to be significantly more likely to experience anxiety symptoms related to COVID-19 than other health workers (AOR: 2.33; 95\% Cl: 1.21-4.47) (Khanal, Devkota, Dahal, Paudel, \& Joshi, 2020). COVID-19 related anxiety can interfere with performance of nurses in their duties and reduce their self-efficacy levels (Pragholapati, 2020). Psychological wellbeing of nurses is crucial for them to effectively discharge their duties. Health care workers including nurses should be afforded opportunities for validating their legitimate anxiety and fears related to COVID-19 (Wong et al., 2020) through periodic screening of COVID-19 related anxiety (Khanal et al., 2020) to promote early intervention. However, in Malawi, nurses are not periodically screened for COVID-19 related anxiety. Therefore, this study aimed at assessing COVID-19 related anxiety among nurses in Malawi.

\section{Methods}

\section{Design}

This was a cross-sectional study that collected data from respondents at one point in time to assess COVID 19 related anxiety among nurses in Malawi.

\section{Setting}

The study setting was Malawi as a country including all members of National Organisation of Nurses in Malawi (NONM) working in Christian Health Association of Malawi (CHAM) and government health facilities.

\section{Study population}

The target population were all Enrolled Nurses, Nurse Midwife Technicians and Registered Nurses in Malawi. Enrolled Nurse and Nurse Midwife Technicians are the lowest cadre of nurses with a college 
Certificate or Diploma in Nursing and Midwifery while Registered Nurses either have Bachelor of Science in Nursing and Midwifery or Bachelor of Science in Nursing plus a University Certificate in Midwifery or University Diploma in Nursing and Midwifery or University Diploma in Nursing plus a University Certificate in Midwifery (Nurses and Midwives Council of Malawi, 2009).

\section{Sample size}

The sample size was calculated using the methodology detailed by Jones, Carley, and Harrison (2003). This was used to ensure there were enough cases and non-cases of anxiety for validating Coronavirus Anxiety Scale. Using estimated Sensitivity $=.90$, estimated Specificity $=.85$ (Lee, 2020), estimated population prevalence of COVID 19 related Anxiety of .23 (Pappa et al., 2020), and width of Confidence Interval 0.05 the following two sample sizes N1 (601) and N2 (255) were calculated. The larger sample size (601) was chosen for this study. However, 320 individuals viewed the survey questionnaire online and a total of 106 respondents completed the questionnaire of which 4 respondent who were not residing in Malawi were excluded resulting in 102 respondents who participated in this study. This was a convenient sample.

\section{Materials}

The data collection instrument for this study included background information, the Coronavirus Anxiety Scale (CAS) and the Work and Social Adjustment Scale (WSAS). The instrument was self-administered in English because nurses are expected to understand English by virtue of their training.

\section{Background information}

Respondents were asked questions related to the background information including: age, gender, level of their education, employment status, marital status, coronavirus diagnosis, history of anxiety, if they are working with a COVID-19 positive patients, if they have a relative or acquaintance with COVID-19 diagnosis (Evren et al., 2020), nursing cadre and workplace.

\section{Coronavirus Anxiety Scale (CAS)}

This study used the Coronavirus Anxiety Scale (CAS) which was specifically designed to assess Anxiety that is triggered by COVID 19 (Lee, 2020). The tool is a 5-item likert scale with each item having 5 possible responses ranging from 0 (not at all) to 4 (nearly every day over the last 2 weeks). The CAS has maximum score of 20 and minimum score of 0 with an optimum cutoff score of $\geq 9$ (Lee, 2020; Lee, Mathis, Jobe, \& Pappalardo, 2020). It is a reliable (Cronbach's Alpha=.93) and valid tool for measuring COVID 19 related Anxiety (Sensitivity $=90 \%$, Specificity $=85 \%$, AUC $=0.94, p<.001)($ Lee, 2020). The instrument was used to distinguish individuals with dysfunctional anxiety and those without anxiety.

Work and Social Adjustment Scale (WSAS) 
This study also used an adapted Work and Social Adjustment Scale (WSAS) to measure functional impairment experienced by respondents (Mundt, Marks, Shear, \& Greist, 2002). The WSAS is 5 -items Likert scale with each item having 9 possible responses ranging from 0 (not at all impaired) to 8 (very severely impaired). The tool has maximum score of 40 and minimum is score of 0 with an optimum cutoff score of $\geq 21$ for moderately severe or worse psychopathology (Lee et al., 2020; Mundt et al., 2002). Scores of 10 to 20 suggests significant functional impairment but less severe clinical symptomatology while scores $<10$ are associated with subclinical populations. It is a reliable instrument (Cronbach's Alpha $\geq .88$ ) (Lee et al., 2020; Mundt et al., 2002). The WSAS was used as a gold standard against which the CAS was validated.

\section{Data collection procedure}

Data collection was conducted from August to September 2020 using a self-administered questionnaire powered by Surveys for Pages and google pages. Online links for the study questionnaire was sent to potential respondents through whatsapp, facebook page, messenger and email. The questionnaire included information about study and a question which asked consent from potential respondents before deciding to participate in the study.

\section{Ethics}

The study received ethics approval and institutional clearance from relevant authorities. The online questionnaire was preceded by an information sheet that explained the nature and benefits of the study to nurses before they were asked to give consent to participate in the study. Respondents' names did not form part of background information that was collected, thus respecting their privacy and maintaining confidentiality. Respondents were informed that only aggregated data will be analysed and disseminated. Respondents were informed that their participation in the study was voluntary and they were free to withdraw at any time if they felt uncomfortable about any aspect during the course of the study. They were also informed that refusing to join the study would not have any effect on their job.

\section{Data analysis}

Data was analysed using Statistical Package for Social Sciences (SPSS) 25. Descriptive statistics including means, standard deviations, percentages, frequencies were used to summarise data for background information, the CAS scores and the WSAS scores. Analysis of variance (ANOVA) and independent samples Students t-tests was used to test for mean differences of respondents scores. A Receiver Operating Characteristic (ROC) analysis was used to generate values for sensitivity, specificity, area under the curve (AUC), positive predictive values (PPV), negative predictive values (NPV) and Youden index for the CAS to identify functionally impaired nurses and test whether or not its original cut-off score of $\geq 9$ (Lee, 2020) remained an optimal score for psychiatric screening in the local setting. Finally, Cronbach's alpha for the CAS and the WSAS were computed to assess their internal consistency locally.

\section{Results}




\section{Prevalence of COVID-19 related anxiety among respondents}

This study found that COVID-19 related anxiety was high $(25.5 \%, n=26)$ among nurses in Malawi. There were significant differences in the numbers of respondents who had COVID-19 related anxiety in relation to workplace $\left(\chi^{2}=8.8, p=0.03\right)$ (Table 1$)$. The prevalence of COVID-19 related anxiety was highest among respondents who were working in hospitals $(36.2 \%, n=21)$ with a mean CAS score of $6.7 \pm 4.8$ (Table 1$)$. The prevalence of COVID-19 related anxiety varied in relation to other demographic characteristics (Table 1). However, this finding was not significant.

\section{Prevalence of functional impairment among respondents}

This study found that nearly half of the respondents $(48 \%, n=49)$ in this study suffered from functional impairement due to COVID-19. There were variations in numbers of respondents who suffered functional impairment in relation to demographic characteristics (Table 2). These differences were not significant $(p>0.05)$ except for gender and workplace. The prevalence of functional impairement was significantly higher among female respondents $(56.2 \%, n=41)$ compared to their male counterparts $(27.6 \%, n=8)$ $\left(x^{2}=6.8, p=0.01\right)$. There were significant differences in the numbers of respondents who had functional impairement in relation to workplace $\left(\chi^{2}=8.7, p=0.03\right)$ (Table 2$)$. The prevalence of functional impairment was highest among respondents who were working in hospitals $(58.6 \%, n=34)$ with mean WSAS score of $20.6 \pm 10.4$ (Table 2). Furthermore, none of those working in COVID-19 ward $(0 \%, n=0)$ had functional impairement with the lowest mean WSAS score of 5.5 \pm 7.8 .

The demographic characteristics which were found to have significant differences based on Chi Square test were further analysed using one-way ANOVA to determine if there were any significant difference in respondent scores on the CAS and the WSAS based on workplace. The findings revealed that there were significant differences in the respondents' mean scores on the CAS $(F(3,98)=3.1, p=0.03)$ and the WSAS $(F(3,98)=3, p=0.03)$ based on workplace. Post hoc comparisons using the Tukey Honestly Significant Difference (HSD) test indicated that the WSAS mean scores for the respondents working in the hospital $(M=20.6 \pm 10.4)$ was significantly different $(p=0.05)$ than those working in Non-Governmental Organisations (NGOs) $(M=13.6 \pm 8.9)$. However, the WSAS mean scores for respondents working in nursing colleges $(M=16 \pm 11.5)$ and COVID-19 wards $(M=5.5 \pm 7.8)$ did not significantly differ from those working in hospital $(p>0.05)$. Independent samples t-tests revealed that female respondents $(M=18.8 \pm 10.4)$ had higher WSAS scores than males $(M=15.9 \pm 11.7)$ and this result was not significant $(t=1.2, p=0$.22). A further, Post hoc comparison using the Tukey HSD test showed that the CAS mean scores for the respondents working in the hospital $(M=6.7 \pm 4.8)$ was significantly different $(p=0.05)$ than those working in NGOs ( $M=2.5 \pm 2.7)$. However, the CAS mean scores for respondents working in nursing colleges $(M=2.8 \pm 4.2)$ and COVID-19 wards $(M=1.5 \pm 0.7)$ did not significantly differ from those working in hospital $(p>0.05)$.

\section{Reliability and Validity of the CAS}


This study revealed that both the CAS (Cronbach's Alpha=0.9) and the WSAS (Cronbach's Alpha=0.8) are reliable instruments with good internal consistency for assessing COVID-19 related anxiety and functional impairment in the local setting. A further analysis using the WSAS as a gold standard, the CAS (cut off $\geq 9$ ) was found to be a valid (Sensitivity $=73.1 \%$; Specificity $=60.5 \%$; Negative Predictive Value $=38.8 \%$; Positive Predictive Value $=86.8 \%$ ) and accurate (AUC=0.73) instrument for measuring COVID-19 related anxiety locally. This study found that the optimum cut off score of the CAS was $>3$ (Youden index $=0.34$ ) which was lower than the original optimum cut off $>9$.

\section{Discussion}

COVID-19 related anxiety is one of the mental health problems affecting nurses. In this study more than a quarter of nurses $(25.5 \%, n=26)$ were found to have COVID-19 related anxiety. This is comparable to prevalence of COVID-19 related anxiety that was found among nurses in China (27.9\%) (Zhu et al., 2020). However, the prevalence of COVID-19 related anxiety found in this study was higher than a pooled prevalence of COVID-19 related anxiety among health workers $(23.2 \%)$ that was reported by a systematic review (Pappa et al., 2020). This may be explained by literature which asserted that nurses usually experience strong emotional reactions to the COVID-19 virus including anxiety which impact on their work (Maben \& Bridges, 2020). In this study almost half of the nurses $(48 \%, n=49)$ had self reported functional impairement related to COVID-19. Mental health problems can significantly reduce quality of care offered by nurses (Puradollah, 2020). This is corroborated by Pragholapati and colleagues who asserted that COVID 19 related anxiety can interfere with performance of nurses in their duties and reduce their selfefficacy levels (Pragholapati, 2020).

This study suggests that many nurses who work in hospitals experience high levels of anxiety $(36.2 \%$, $\mathrm{n}=21$, Mean CAS score $=6.7 \pm 4.8)$ and functional impairment $(58.6 \%, \mathrm{n}=34$, Mean WSAS score $=20.6 \pm 10.4)$. As front-line health care workers, nurses may be vulnerable to negative mental health effects from COVID-19. The nature of care itself and new ways of working are potentially highly stressful for nurses who are being overwhelmed with increased workload and demands to accommodate new protocols (Maben \& Bridges, 2020). Literature indicated that it is necessary to pay attention to the psychological issues of nurses during and after caring for patients with COVID-19 (Puradollah, 2020). Supporting nurses practically and psychologically helps in preserving their health especially when workrelated stress levels are so high (Maben \& Bridges, 2020).

Hospitals should provide sufficient support to nurses including personal protective equipment, psychological screening for nurses and psychological support.(Puradollah, 2020). This is crucial because nurses may not be able to recognise and deal with their own mental health problems while they provide care to COVID-19 patients. This is supported by a body of literature which suggested that those injured by stress may be the last to recognise it and individuals often do not prioritise taking good care of themselves (Maben \& Bridges, 2020). It is necessary that nurses with symptoms of anxiety seek help from psychotherapists to evaluate them and help them deal with potential stress (Huang, Lin, Tang, Yu, \& Zhou, 2020). This is helpful because nurses must be able to look after themselves if they are going to 
properly look after others (Maben \& Bridges, 2020). However, nurses are generally trained to look after others and not self so that in many instances they need others including colleagues, friends and managers to remind them to think of themselves (Maben \& Bridges, 2020).

Reliable and valid instruments are needed for nurses to detect and validate their COVID-19 related anxiety. Due to a concern about the variation of performance of screening instruments in different populations and settings (Bossuyt et al., 2015), it was necessary to measure validity of the CAS in the local setting. This study showed that the CAS was able to distinguish nurses with anxiety and those without anxiety (Sensitivity=73.1\%; Specificity $=60.5 \%$; AUC $=0.73$; cut off $\geq 9$ ) in the local setting. The results support the CAS as an effective, reliable (Cronbach's Alpha $=0.9$ ) and valid instrument for clinical research and practice (Evren et al., 2020; Lee, 2020; Lee et al., 2020).

This study had some limitations because it was conducted online and those without Internet access or without adequate internet bundles could not be involved in the study. Furthermore, the results of this study heavily relied on respondents' self-reports which may have yielded recall bias because nurses were asked to recall events that happened some two weeks or more ago. There was no common attention check item (instructed response item) embedded within the online survey. Consequently, the validity of the CAS might have been affected by careless responses.

\section{Conclusion}

This study suggested that prevalence of COVID-19 related anxiety is high among nurses in Malawi. Most nurses experience functional impairment because of COVID-19. Furthermore, this study has confirmed that the CAS is a valid instrument which is effective in detecting COVID-19 related anxiety among nurses. The CAS could be used for diagnosis in clinical practice and in developing prevention programs in busy settings because it can be administered quickly for it only has five items (Evren et al., 2020).

\section{IMPLICATIONS FOR PRACTICE}

Considering that prevalence of COVID-19 related anxiety is high among nurses, it is necessary to screen them for the condition using the CAS and provide them effective psychosocial interventions. Psychological interventions targeting health care workers particularly nurses, should be a part of preparedness to reduce COVID-19 impact on their well-being and the health system at large (Khanal et al., 2020). Nurses need their employers, their teams, the profession and the public to support them with action and resources (Maben \& Bridges, 2020). They need access to rest breaks, good peer and team support and leaders that will continue to care for them well after the pandemic is over. The psychosocial interventions for nurses should include a place to rest; provision of food and daily living supplies; training to manage patient's psychological problems; and access to psychological counsellors who can listen to nurses' difficulties and provide support accordingly (Maben \& Bridges, 2020).Furthermore, the COVID-19 crisis should provide the impetus for the government to place more emphasis on the allocation of financial resources to mental health services and staff support programmes (Cheung, Fong, \& Bressington, 2020) for nurses. 


\section{References}

1. Ahorsu, D. K., Lin, C.-Y., Imani, V., Saffari, M., Griffiths, M. D., \& Pakpour, A. H. (2020). The fear of COVID-19 scale: development and initial validation. International Journal of Mental Health and Addiction.

2. Cheung, T., Fong, T. K., \& Bressington, D. (2020). COVID-19 under the SARS Cloud: Mental Health Nursing during the Pandemic in Hong Kong. Journal of psychiatric and mental health nursing.

3. Evren, C., Evren, B., Dalbudak, E., Topcu, M., \& Kutlu, N. (2020). Measuring anxiety related to COVID19: A Turkish validation study of the Coronavirus Anxiety Scale. Death studies, 1-7. doi:10.1080/07481187.2020.1774969

4. Huang, L., Lin, G., Tang, L., Yu, L., \& Zhou, Z. (2020). Special attention to nurses' protection during the COVID-19 epidemic. BioMed Central, 24, 120.

5. Jones, S., Carley, S., \& Harrison, M. (2003). An introduction to power and sample size estimation. Emergency Medicine Journal: EMJ, 20(5), 453.

6. Khanal, P., Devkota, N., Dahal, M., Paudel, K., \& Joshi, D. (2020). Mental health impacts among health workers during COVID-19 in a low resource setting: a cross-sectional survey from Nepal. 1-27.

7. Lee, S. A. (2020). Coronavirus Anxiety Scale: A brief mental health screener for COVID-19 related anxiety. Death studies, 44(7), 393-401.

8. Lee, S. A., Mathis, A. A., Jobe, M. C., \& Pappalardo, E. A. (2020). Clinically significant fear and anxiety of COVID-19: A psychometric examination of the Coronavirus Anxiety Scale. Psychiatry research, 113112 .

9. Maben, J., \& Bridges, J. (2020). Covid-19: Supporting nurses' psychological and mental health. Journal of clinical nursing, 29, 2742-2750.

10. Mundt, J. C., Marks, I. M., Shear, M. K., \& Greist, J. M. (2002). The Work and Social Adjustment Scale: a simple measure of impairment in functioning. The British Journal of Psychiatry, 180(5), 461-464.

11. Nelson, S. M., \& Lee-Winn, A. E. (2020). The mental turmoil of hospital nurses in the COVID-19 pandemic. Psychological Trauma: Theory, Research, Practice, and Policy, 12(1), 126-S127.

12. Nurses and Midwives Council of Malawi. (2009). Scope of Practice for All Cadres of Nursing and Midwifery. Mzuzu: Katoto Printing Press.

13. Pappa, S., Ntella, V., Giannakas, T., Giannakoulis, V. G., Papoutsi, E., \& Katsaounou, P. (2020). Prevalence of depression, anxiety, and insomnia among healthcare workers during the COVID-19 pandemic: A systematic review and meta-analysis. Brain, Behavior, and Immunity. doi:https://doi.org/10.1016/j.bbi.2020.05.026

14. Pragholapati, A. (2020). Self-Efficacy Of Nurses During The Pandemic Covid-19 (pp. 1-7): no.

15. Puradollah, M. (2020). Necessity of Attention to Mental Health of the Front Line Nurses against COVID-19: A Forgotten Requirement. International Journal of Community Based Nursing and Midwifery, 8(3), 280. 
16. Shanafelt, T., Ripp, J., \& Trockel, M. (2020). Understanding and addressing sources of anxiety among health care professionals during the COVID-19 pandemic. JAMA, 323(21), 2133-2134.

17. Wong, A. H., Pacella-LaBarbara, M. L., Ray, J. M., Ranney, M. L., \& Chang, B. P. (2020). Healing the Healer: Protecting Emergency Health Care Workers' Mental Health During COVID-19. Annals of Emergency Medicine, 1-6. doi:https://doi.org/10.1016/j.annemergmed.2020.04.041

\section{Tables}

Due to technical limitations, tables 1-2 are only available as a download in the supplemental files section.

\section{Supplementary Files}

This is a list of supplementary files associated with this preprint. Click to download.

- Tables.pdf 